\title{
Real-time network simulation support for scalable routing experiments
}

\section{Yue Li*, Jason Liu and Raju Rangaswami}

School of Computing and Information Sciences,

Florida International University,

Miami, Florida 33199, USA

E-mail: yueli@cis.fiu.edu

E-mail: liux@cis.fiu.edu

E-mail: raju@cis.fiu.edu

${ }^{*}$ Corresponding author

\begin{abstract}
This paper describes a new software infrastructure that combines the scalability and flexibility benefits of real-time network simulation with the realism of open-source routing protocol implementations. The infrastructure seamlessly integrates the open-source XORP router implementation with a real-time large-scale network simulation engine. The design uses a novel forwarding plane offloading approach that decouples routing from forwarding and confines the more resource consuming forwarding operations inside the simulation engine to reduce I/O overhead. Experiments demonstrate superior performance of the software routing infrastructure without impairing accuracy. The infrastructure is shown to be able to support large-scale routing experiments on light-weight virtual machines.
\end{abstract}

Keywords: network simulation; real-time simulation; network emulation; routing protocols; large-scale routing experiments.

Reference to this paper should be made as follows: Li, Y., Liu, J. and Rangaswami, R. (2009) 'Real-time network simulation support for scalable routing experiments', Int. J. Simulation and Process Modelling, Vol. 5, No. 2, pp.146-156.

Biographical notes: Yue Li is a Postdoctoral Research Associate in the School of Computing and Information Sciences, Florida International University. He received PhD Degree in Computer Science from Xi' an Jiao Tong University, China in 2005. He joined Tsinghua National Laboratory for Information Science and Technology as a postdoc from 2005 to 2007. His research interests include network simulation and emulation, high performance simulation, and multimedia networking.

Jason Liu is an Assistant Professor in the School of Computing and Information Sciences, Florida International University. His research focuses on parallel discrete-event simulation, performance modelling and simulation of computer systems and communication networks. He received a BA in Computer Science from Beijing University of Technology in China in 1993, an MS in Computer Science from College of William and Mary in 2000, and a PhD in Computer Science from Dartmouth College in 2003.

Raju Rangaswami is an Assistant Professor of Computer Science at the Florida International University in Miami. He received a BTech Degree in Computer Science from the Indian Institute of Technology, Kharagpur, India. He obtained MS and PhD Degrees in Computer Science from the University of California at Santa Barbara. His research interests include operating systems, storage systems, virtualisation, security, and real-time systems.

\section{Introduction}

Researchers are continuously searching for techniques that can better evaluate the dynamic behaviour of routing protocols in complex, large-scale network scenarios. Such techniques can help evolve our understanding of complex network problems, such as the stability issues with BGP and OSPF (Basu and Riecke, 2001; Shaikh et al., 2000, 2002; Shaikh and Greenberg, 2001), BGP convergence (Obradovic, 2002), and BGP security and misconfiguration (Goodell et al., 2003; Chim and Yeung, 2004; Mahajan et al., 2002; Nordstrom and Dovrolis, 2004). Existing techniques for understanding and evaluating network routing protocols can be categorised as analytical, simulation-based, and emulation-based. Here we exclude direct experimentation on physical networks, 
because it is infeasible to conduct custom large-scale routing experiments directly on routers deployed deep in the internet.

Analytical techniques, which can often bring valuable insight to the design of large-scale complex systems, are ill-equipped to address the complexities and dynamics inherent with the actual protocol execution. Simulation is used commonly by researchers to perform large-scale routing experiments since this technique can capture the network dynamics with a great amount of detail. Bauer et al. (2006), Liu and Chien (2004) and Dimitropoulos and Riley (2004). However, a simulation model of a router's behaviour can be quite different from a real routing protocol implementation and thus may not provide the necessary realism of protocol execution behaviour. Further, simulation implementation of routing protocols typically comes with only a modest selection of protocols and protocol versions, thus limiting its utility.

Emulation-based techniques allow the execution of network routing protocols 'as is' within an emulated network environment, providing the necessary realism in the protocol execution. With the emergence of open-source router software, such as XORP (2008), Zebra (2008) and Quagga (2008), researchers can now prototype and evaluate routing protocols on actual systems as part of the emulation testbed. Emulation-based techniques, however, must address the issues of scalability and flexibility. The scalability of an emulation testbed is constrained by the available physical resources. For example, experimental network scenarios must observe the limits on bandwidth, latency, and node counts of the underlying physical infrastructure. Emulation-based solutions are thus incapable of creating arbitrary network topologies and controlling background network traffic conditions.

Our position is that the design of a successful framework for routing experiments must comprehensively address the three challenges of realism, scalability, and flexibility. In this paper, we describe an infrastructure that we have built to support scalable routing experiments based on a real-time network simulation system. A key advantage of our approach is that, since it provides a real-time communication interface to real client machines, commodity applications can be executed unmodified within this framework (Liu, 2008). Consequently, if engineered correctly, such a simulation framework can provide a realistic network infrastructure indistinguishable from a real network from the standpoint of the real applications. When this framework is coupled with machines running open-source router software, it can provide a realistic, scalable, and flexible infrastructure for conducting routing experiments. We address the three challenges comprehensively as we describe below:

- $\quad$ Realism. Our infrastructure uses a previously developed real-time network simulator, called PRIME (2008). PRIME provides an emulation infrastructure which supports a seamless integration of a large number of prototype network applications or protocols into the experimental environment. The infrastructure we have developed integrates PRIME with the open-source (XORP, 2008) router software, making it possible to conduct large-scale routing experiments. This technique also achieves a high level of realism since actual network protocol implementations are directly included into the experiments.

- Scalability. Traffic on the virtual network must be forwarded based on routing decisions made by XORP instances. If each packet must be exported from the simulator and sent to an XORP router instance, and then immediately received and imported back into the simulator as soon as forwarding is achieved at the router, the $\mathrm{I} / \mathrm{O}$ overhead would be substantial and might limit the scale of the routing experiments. We propose a forwarding plane offloading approach, which allows the XORP router to designate the packet forwarding function to the simulator and communicates with the simulator its routing decisions through an out-of-band channel. In doing so we can confine the background traffic (unrelated to routing) within simulation, thereby eliminating the I/O overhead associated with the bulk of the network traffic. Furthermore, since packet transmissions can be parallelised somewhat easily, we are able conduct routing experiments with a network size far beyond what can be supported by emulation testbeds.

- Flexibility. It is straightforward to apply real-time simulation to explore a wide spectrum of network scenarios and address what-if questions (e.g., by injecting network failures). Real-time simulation is based on simulation. Once a simulation model is developed, reasonably verified and validated, it takes little effort to conduct simulation experiments to cover a large parameter space. The proposed infrastructure also allows incorporating analytical models in the real-time network simulation.

For example, a user of the infrastructure can employ low-resolution models to describe aggregate internet traffic (e.g., Liu, 2006) and thus considerably increase the size of the network under test. Further, it is relatively easy to manipulate and control dynamic network behaviour with simulation. For instance, the user can bring down a link in the virtual network and observe the reaction mechanism of the routing protocols.

\section{Background}

Our infrastructure for scalable routing experiments builds on top of a real-time parallel simulation engine called PRIME, which stands for a Parallel Real-time Immersive Modelling Environment (PRIME, 2008). PRIME enables real-time large-scale network simulations and supports seamless interactions with real distributed applications. 
PRIME is built on top of a parallel discrete-event simulation kernel that implements the Scalable Simulation Framework (SSF) (Cowie et al., 1999). The parallel simulation kernel deals with synchronisation and communication issues between logical processes that execute simulation events in parallel, while PRIME itself enables the notion of real time by incorporating packets from external application as real-time events. In order to meet the deadline requirement of the real-time events, PRIME employs a greedy priority-based scheduling algorithm - real-time events are assigned with a higher priority to ensure their timely processing.

PRIME can interact with a large number of clients, which may be traffic sources, sinks, or other network entities like routers. It is important to be able to incorporate a large number of such clients and yet remain transparent to the applications running on the clients. There are several ways to incorporate real applications as part of the simulation environment, which include using packet capturing techniques (such as libpcap, IP table, and IP tunnel), preloading dynamic libraries, and modifying the binary executables, and so forth. PRIME instead uses an open systems approach based on a Virtual Private Network (VPN) solution (Liu et al., 2007). We customise VPN to function as a gateway that bridges traffic between the clients and the simulated network. Clients establish connection to the simulation gateway. Traffic generated by the clients and destined for the virtual network is directed by the modified VPN through the gateway to the real-time network simulator.

We illustrate this approach using an example. Suppose we have two BGP routers which need to build up the peering relationship with each other. VPN sets up two TUN interfaces with IP addresses of 10.10.0.2 and 10.10.1.8 for one router, similarly 10.10 .1 .9 and 10.10 .1 .20 for another router. The corresponding simulated nodes have the same IP address configuration, as shown in Figure 1. One BGP speaker first sends an OPEN message with destination IP address of 10.10.1.9 via its interface 10.10.1.8, then the packet is sent to the modified VPN server at the simulation gateway through a VPN connection. The simulation gateway forwards the packet to PRIME via a dedicated TCP connection. In PRIME, the packet is treated as a real-time event and injected into the simulation event queue. PRIME simulates the packet being forwarded on the virtual network as if it was created by the simulated node with the same IP address 10.10.1.8. Upon reaching the node 10.10.1.9, the packet will be exported from simulation engine, travelling in the reverse direction via the simulation gateway back to the external router with the IP address 10.10.1.9.

A distinct advantage of this approach is that the emulation infrastructure does not require special hardware to set up. It is also secure, a merit inherited directly from the underlying VPN implementation, and scalable, since clients need not be co-located within the simulation infrastructure. Further, multiple simulation gateways can be used simultaneously to accommodate larger traffic capacity between the clients and the real-time network simulator. In order to produce accurate results, however, the emulation infrastructure needs a tight coupling between the emulated entities (i.e., the clients) and the real-time simulator. In particular, the segment between the clients and the real-time network simulator needs to include low-latency links. To maintain potentially high traffic throughput demand, this segment must also have sufficient bandwidth for tunnelling traffic through the emulation infrastructure.

In the next section, we develop techniques that allow the above real-time simulation infrastructure to scale up when used specifically for network routing experiments.

Figure 1 The emulation infrastructure based on VPN (see online version for colours)

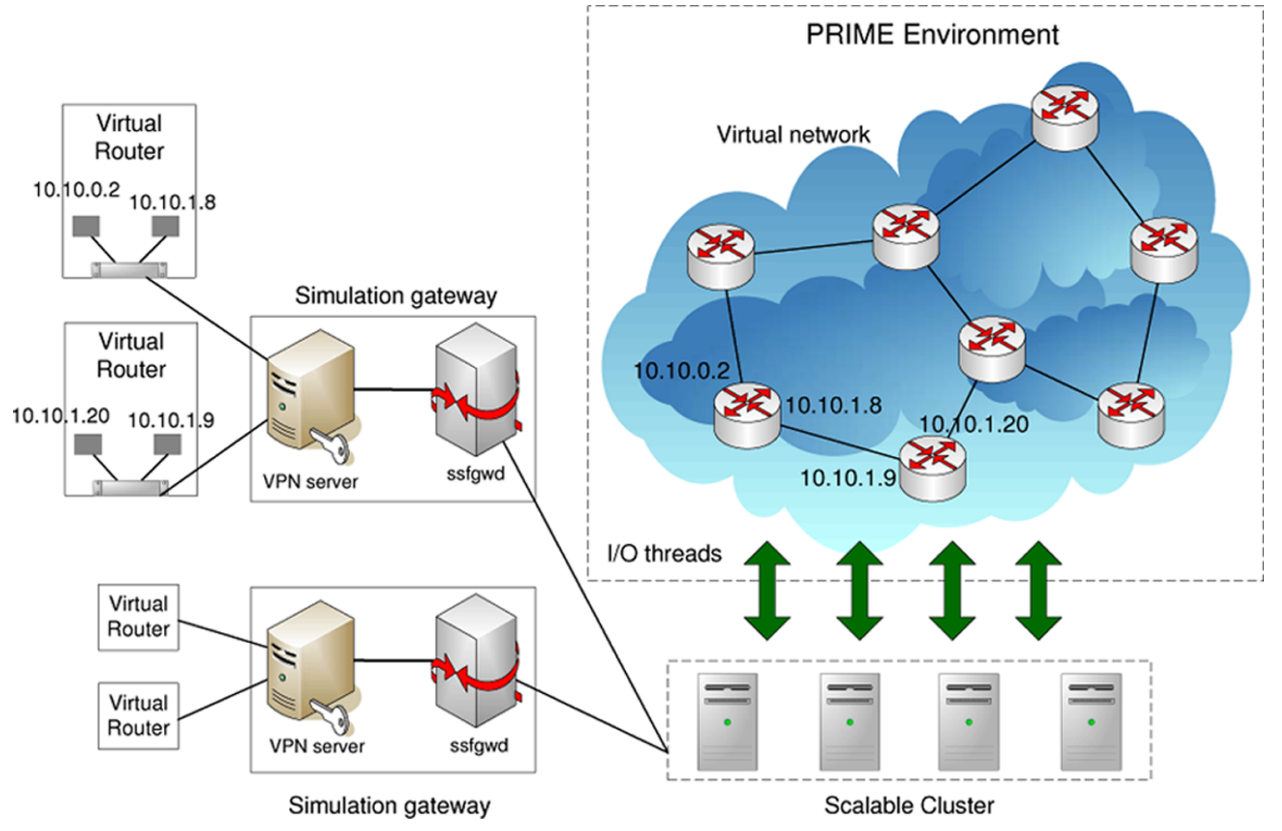




\section{Scalable routing infrastructure}

The availability of open-source router platforms, such as XORP, Zebra, and Quagga, has simplified the task of researchers, who can now prototype and evaluate routing protocols with relative ease. To support experiments on a large-scale network consisting of many routers with multiple traffic sources and sinks, we propose to integrate the open-source router platforms with a real-time network simulation infrastructure.

\subsection{Virtual router}

In order to scale up the experiment in an easy way we adopt virtualisation technology to host multiple router instances. Virtualisation helps maintain isolated namespaces, each of which manages the virtual resources necessary for executing a router instance. We therefore call each instance a virtual router. Specifically, four types of network resources shall be provided within a virtual router: network sockets, network interfaces, forwarding table, and loopback device. Routing protocols, e.g., BGP, RIP, and OSPF, need to send and receive packets via standard network sockets (TCP, UDP, and raw sockets) in order to establish peering connectivity and exchanging routing information. Network interfaces and the forwarding table together represent the network forwarding engine of the virtual router on which routing protocols are run. A network loopback device is sometimes used by the routing protocols to communicate among separate processes (e.g., see XORP, 2008).

In particular, we investigate four popular virtualisation technologies: Xen (Barham et al., 2003; OpenVZ, 2008), Linux-VServer (Soltesz et al., 2007) and VRF (2008). As we can see in Table 1, all four types of network resources are provided in Xen and OpenVZ, while Linux-VServer and VRF have only partial network virtualisation support. For this reason, we focus only on Xen and OpenVZ in our experiment studies.

Table 1 Network virtualisation in Xen, OpenVZ, Linux-VServer and Linux-VRF

\begin{tabular}{lcccc}
\hline & Xen & OpenVZ & VServer & VRF \\
\hline Network sockets & $\checkmark$ & $\checkmark$ & $\checkmark$ & $\checkmark$ \\
Network interfaces & $\checkmark$ & $\checkmark$ & & $\checkmark$ \\
Forwarding table & $\checkmark$ & $\checkmark$ & \\
Loopback device & $\checkmark$ & $\checkmark$ & \\
\hline
\end{tabular}

We also examined the scalability aspects of these network virtualisation choices. We found that the network stack in Xen (version 3.0.4) becomes unstable under high traffic load with 32 or more domains. This phenomenon is consistent with similar observations in other studies (Padala et al., 2007; Bhatia et al., 2008). As a result, we choose OpenVZ as the platform for our large-scale routing experiments.

\subsection{Forwarding plane offloading}

PRIME provides real-time simulation capabilities and an emulation infrastructure that can seamlessly integrate multiple clients, may they be routers or otherwise. Since the routers must be emulated outside PRIME on client machines where they can run the real routing software directly, every packet travelling along its path from the source to the destination must be exported to each intermediate router for forwarding decisions, and subsequently imported back into the PRIME simulation engine. Thus, the forwarding operation for each packet at each hop incurs a substantial I/O overhead. Consequently, the overall overhead will significantly impact the performance of the infrastructure, especially in large-scale routing experiments. To avoid this problem, we propose a forwarding plane offloading approach, which moves the packet forwarding functions from the router software to the simulation engine. Since packet forwarding operations are carried out entirely with PRIME, we can eliminate the I/O overhead associated with communicating bulk-traffic back and forth between the router software and the real-time simulator.

This approach, however conceptually straightforward, raises two key design questions. First, the forwarding table for a specific virtual node used by the simulator for internal forwarding decisions must be synchronised with the actual forwarding table, which is updated by the routing protocols running within the corresponding external router instance. The synchronisation is critical to ensure correct forwarding operations within the simulation engine. Second, the simulation engine must be instantly informed of any network interface reconfigurations performed at the external router instance. For instance, a network administrator may create virtual network interfaces or reset the MTU of an existing network interface; in such cases, the corresponding simulated router node must be kept consistent with the changes.

\subsection{The XORP architecture}

In our current implementation, we combine the open-source XORP router software with PRIME to provide a scalable platform for conducting routing experiments. Two reasons prompted our choice of XORP. First, XORP has the most up-to-date implementations of routing protocols, including those commonly used protocols, such as BGP, OSPF, and RIP; it is operation is consistent with real router software and it is a well-established experimental platform for routing protocol design and evaluation by the routing protocol research community. Second, XORP implements a Forwarding Engine Abstract (FEA) layer, which splits the routing and forwarding plane as two separate layers within its implementation, thus considerably simplifying our task of offloading the latter to PRIME.

The primary role of FEA is to shield XORP processes from concerns of forwarding plane operations, so that arbitrary forwarding mechanisms can be employed 
via plug-ins. FEA has three main components: Forwarding Information Base (FIB) manager, interface manager, and $\mathrm{I} / \mathrm{O}$ manager. These components are depicted in Figure 2, along with the corresponding XORP processes that interact with FEA. The FIB manager receives forwarding table updates from the Routing Information Base (RIB) process, which arbitrates route updates among routing protocols (e.g., BGP or OSPF). The FIB manager propagates the necessary changes (such as inserting or deleting routing entries) to the underlying forwarding engine. Currently, XORP can choose either the traditional Unix kernel (via Netlink) or the Click modular software router (Kohler et al., 2000) for forwarding. Access to the forwarding plane is performed by the corresponding plug-ins.

Figure 2 Offloading the forwarding plane to the PRIME simulation environment (see online version for colours)

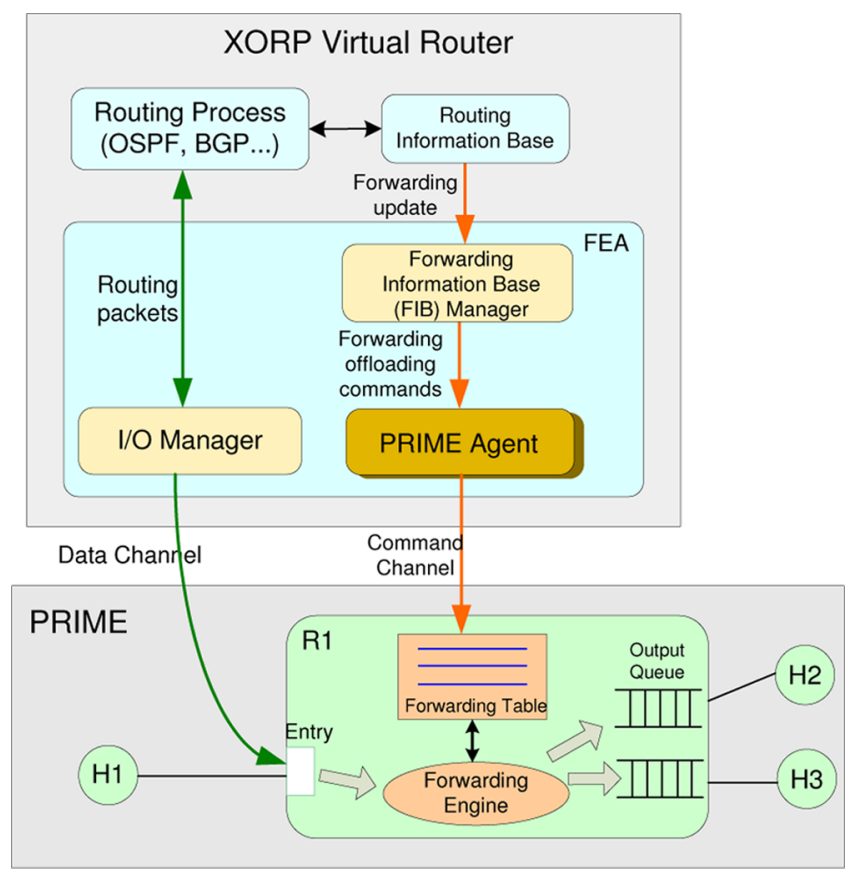

The router manager process can also issue interface configuration requests to the FEA interface manager, according to a pre-specified router configuration file or real-time input from the system administrator via a command-line interface. FEA interprets and executes these requests on behalf of the underlying forwarding engine. Individual routing processes can register with FEA to be notified of changes in the interface configuration. The I/O manager also provides an interface for all the routing protocols to communicate routing information (such as OSPF's Link State Update messages) with their peers on other router instances.

\subsection{XORP forwarding plane offloading implementation}

Figure 2 describes our architecture for forwarding plane offloading with XORP. We create an additional XORP forwarding plane plug-in within FEA, which we call the PRIME Agent. It consists of two components: the PRIME Socket and the Interface Agent. Both components maintain a common command channel with the PRIME simulator for transferring forwarding information updates (via the PRIME Socket) and interface configuration requests (via the Interface Agent) to the corresponding router node in PRIME. This effectively results in the forwarding functionality being offloaded from XORP to PRIME; traffic on the simulated network can thus be kept within simulation.

The interaction between the PRIME Agent and the simulation mechanism is managed as follows. The PRIME Socket provides primitives to manage the forwarding table in PRIME, including those that add or remove a single forwarding entry as well as the entire forwarding table. The PRIME Socket accepts a command from the FIB manager, packages the command into a control packet, and sends it through the command channel. We designate an unused address as the destination address of the control packets, so that they can be distinguished from regular data packets when reaching the simulator through the emulation infrastructure. At the PRIME-end of the command channel, the control packets are translated into simulation events and presented to the emulation protocol session at the corresponding router node. The emulation protocol session interprets the control commands and executes them by modifying the forwarding table on the router node as directed by XORP. The procedure of interface management is essentially the same. The interface manager can modify the network interface configuration at the corresponding router node through primitives provided by the Interface Agent of the FEA plug-in.

Since forwarding table updates and interface management requests are propagated to the simulation engine, the forwarding decisions inside simulation are synchronised with the XORP instances, except when the update commands are in transit. (We investigate the possible discrepancy in Section 4.3). The routing messages created by the XORP routing protocol instances are treated as foreground traffic from the perspective of PRIME and they are forwarded through the simulation engine with the aid of the emulation infrastructure as described in Section 2. This mechanism is enabled by independent data channels on top of VPN automatically.

\section{Experiments}

We conducted three sets of experiments to evaluate our infrastructure for performance, accuracy, and scalability. The initial set of experiments (Section 4.1) were controlled microbenchmarks that allow fine-grained evaluation of the proposed infrastructure, both with and without forwarding plane offloading to the simulator. Next, we carried out an intra-domain routing experiment of a real network topology (Section 4.2) to demonstrate the capability of our infrastructure. Finally we conducted 
scalability studies to explore how large an experiment can be supported by our routing infrastructure.

\subsection{Microbenchmarks}

Since PRIME needs to interact with the XORP instances and simulate the virtual network in real time, the timing accuracy is paramount in obtaining trustworthy experimental results. This means that the infrastructure (both PRIME and the external XORP router instances) should keep up with the wall-clock time, receive real-time events promptly and output them without missing their real-time deadlines. The purpose of our microbenchmark-based evaluation was to illustrate that the proposed infrastructure can support scalable and real-time routing experiments with the forwarding plane offloading technique.

We used an extensible network model for microbenchmarking, as shown in Figure 3. Node $S$ and $C$ acted as the server and client, respectively; the server initiated constant-bit-rate UDP traffic to the client. The intermediate nodes, $R_{1}, R_{2}, \ldots, R_{n}$, were routers. Each router node had one corresponding XORP instance running outside PRIME. We configured each XORP instance to run a OSPFv2 protocol.

Figure 3 Model topology used for microbenchmark experiments (see online version for colours)

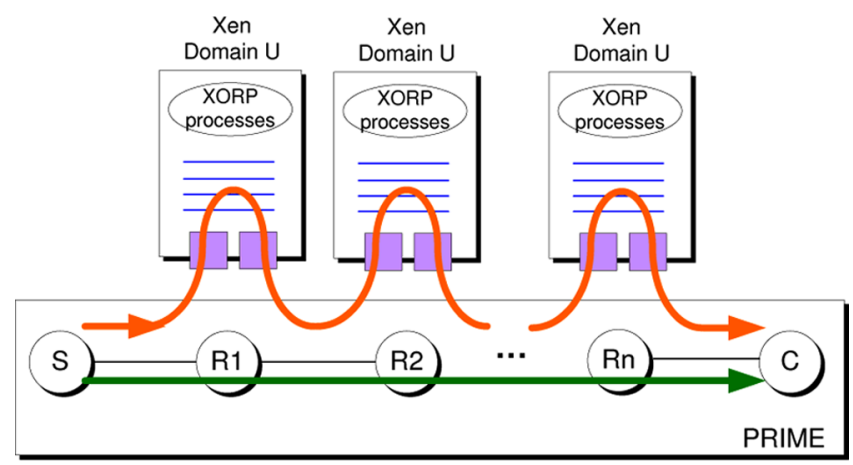

Xen Domain0

For the microbenchmark experiment we adopted Xen virtual machines (Barham et al., 2003) to host PRIME and XORP instances. In Xen terminology, each domain is a virtual machine running on the host machine. Domain 0 is the first domain created when the host is powered on and has privileges to access physical machine resources. It also provides an interface to manage other domains (called Domain U's) run unprivileged.

We set up the Xen environment on a DELL OPTIPLEX 745 workstation with Intel Core 2 Duo $2.4 \mathrm{GHz}$ processors and 4 GB memory. Both the host OS and guest OS ran Debian Linux 4.0 distributions with kernel version 2.6.16.33. PRIME and the simulation gateway ran in Xen Domain 0 and each Domain $U$ contained one XORP instance. We wrote a set of scripts to support rapid deployment of an arbitrary network experiment scenario.

To evaluate performance of the simulation infrastructure and its impact on timing fidelity, we measured round-trip delay and throughput as the primary metrics for evaluation. We evaluated two settings of the simulation infrastructure implementation: offloading-disabled (in which the forwarding plane offloading optimisation was not used) vs. offloading-enabled (in which the optimisation was employed).

Figure 4 depicts the round-trip delay measured by ping between the client and the server as we increased the number of intermediate router hosts running XORP. The propagation delay of individual links were set to be $10 \mathrm{~ms}$ each. Consequently, the ideal round trip time would be $(2 \times 10 \times n)$, where $n$ is the number of intermediate routers. We varied the UDP background traffic between the server and the client to be either $1 \mathrm{Mb} / \mathrm{s}$ or $2 \mathrm{Mb} / \mathrm{s}$. When we disabled forwarding plane offloading, the observed round-trip times deviated from the ideal case, especially when the number of intermediate XORP instances was large. The two curves representing the cases with offloading disabled overlap. The observed values deviated by as much as $30 \%$ with 32 XORP instances. With $2 \mathrm{Mb} / \mathrm{s}$ offered load, the variation of the round-trip time was slightly larger. Note that the link bandwidths were set to $100 \mathrm{Mb} / \mathrm{s}$, which was substantially larger than the offered load, and consequently should not noticeably affect the round-trip times in the ideal case. The observed values when offloading was enabled matched almost exactly with the ideal values, with less than $0.2 \%$ error, regardless of the offered load.

Figure 4 Impact on round-trip delays

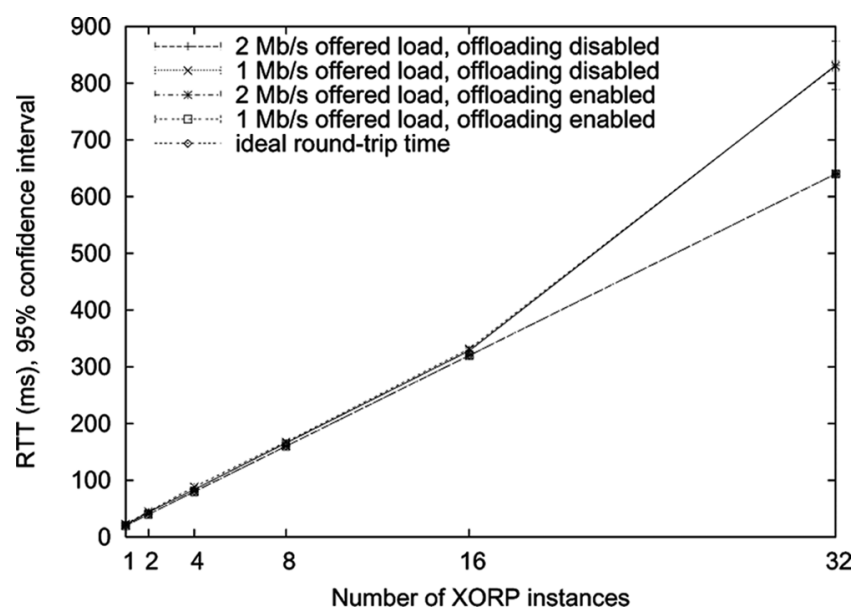

Figure 5 depicts the effect of the simulation infrastructure overhead on traffic throughput between the client and server nodes, again as we varied the number of intermediate XORP router instances. The throughput is calculated by the total size of data transferred from the server to the client divided by the time since the download request is made by the client. The throughput decreased slightly (even in the idea case) with more router instances and therefore longer path between the server and the client. With offloading disabled, the observed throughput was lower than that of the ideal case, becoming more apparent with more intermediate XORP instances. With offloading enabled, 
the observed throughput deviated only slightly (less than $3 \%$ with 32 XORP instances). As expected, since the data download traffic was routed within simulation, rather than being forwarded through the emulation infrastructure, we achieved much better accuracy.

Figure 5 Impact on throughput

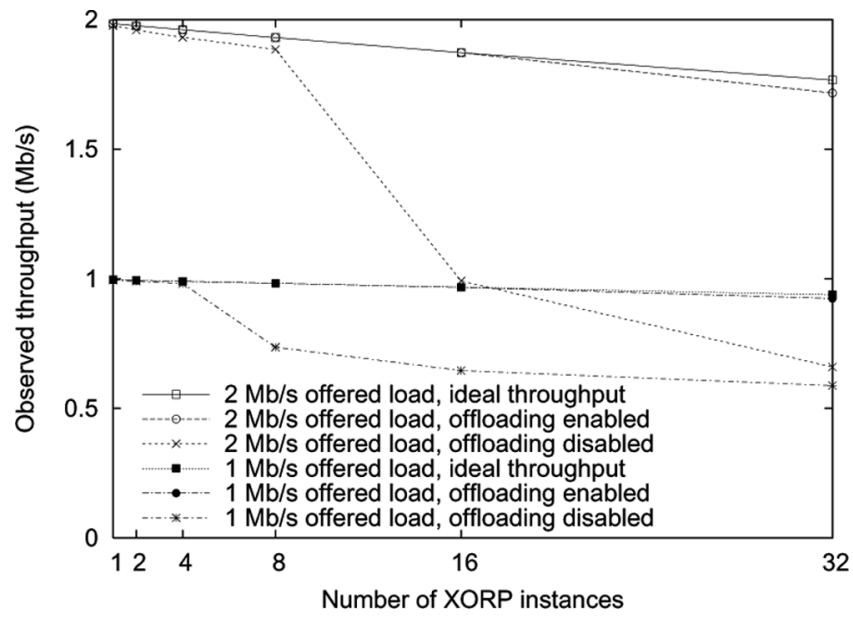

\subsection{An intra-domain routing experiment}

In this section we describe an intra-domain routing experiment as a case study to demonstrate that a realistic routing experiment can be achieved with the integration of PRIME and XORP. The experiment was originally used by VINI (Bavier et al., 2006) to demonstrate its capability to conduct elaborate routing tests on the PlanetLab. The experiment consisted of a realistic Abilene network model with 11 routers, as shown in Figure 6. We configured the model using the real topology, with link delays and bandwidths obtained from the observatory data available at Abilene Observatory Web Site (Abilene Network, 2008). In particular, our model reflected a snapshot of the Abilene backbone on 14, January 2008 at 07:45:11 UTC. We also configured each XORP instance to run OSPFv2 protocol. We used the same Xen virtual machine environment as described in Section 4.1 for the experiment setup.

Figure 6 The Abilene network (see online version for colours)

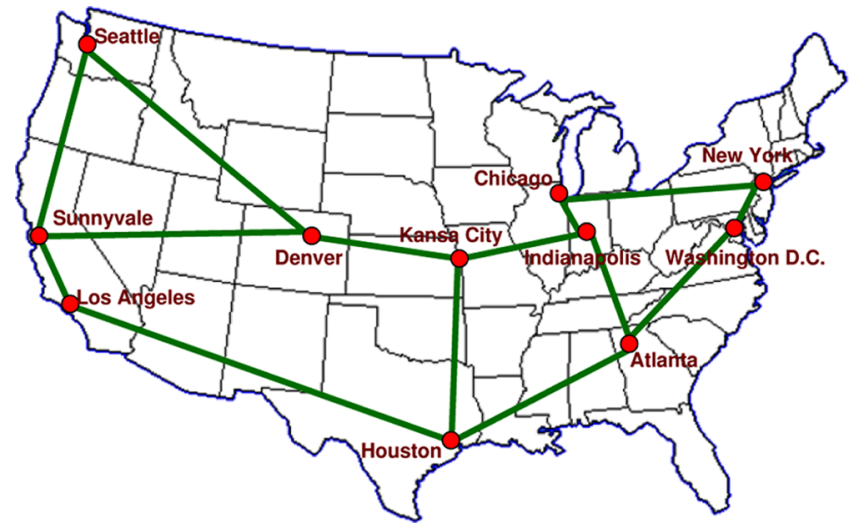

The purpose of the experiment is to observe the convergence of OSPF and its effect on data traffic. We injected a link failure, followed by a recovery between the routers at Chicago and Indianapolis (via the XORP command-line interface). We measured the effect on the round-trip time and data throughput between Denver and Chicago. Figure 7 shows the round-trip time measured by ping. When the link went down at $14 \mathrm{~s}$, OSPF instances recomputed the routes resulting a diversion of the traffic from the upper links to the lower ones. The round-trip delays were increased from around $36 \mathrm{~ms}$ to around $60 \mathrm{~ms}$. The link was later repaired at $43 \mathrm{~s}$ into the experiment. After the routes converged, OSPF re-established the shortest path between the two cities.

Figure 7 OSPF route convergence indicated by ping RTT

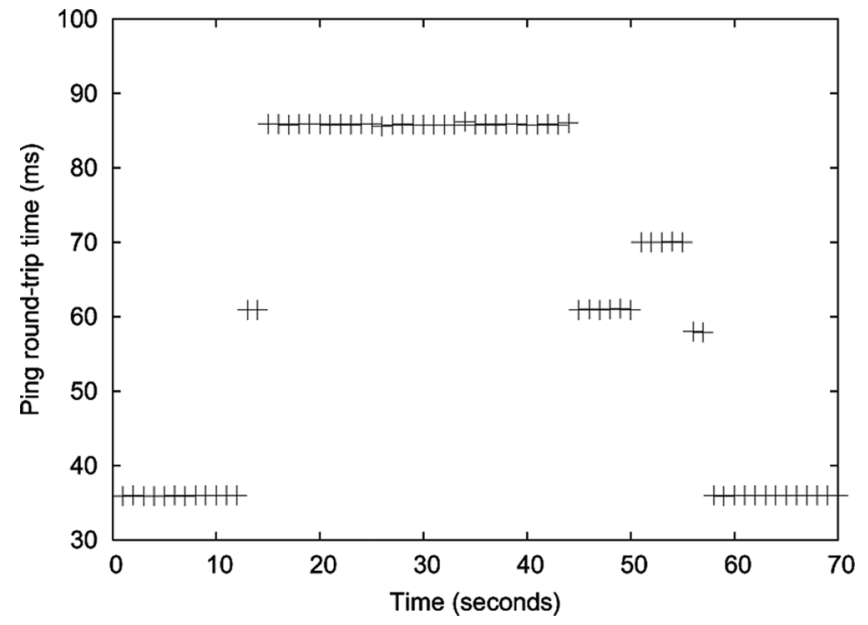

We also used iperf to initiate a TCP transfer from Denver to Chicago in a separate run and analysed the tcpdump results. Figure 8 shows the number of bytes received at the receiver end (i.e., the router at Chicago). The TCP transfer was interrupted when link failure occurred at $18 \mathrm{~s}$ and then resumed when an alternative route was found (at $32 \mathrm{~s}$ ). At $50 \mathrm{~s}$, the link was repaired, the shortest path was re-established, and a better throughput was achieved (demonstrated by a steeper slope in the figure).

Figure 8 TCP transfer during link failure and recovery

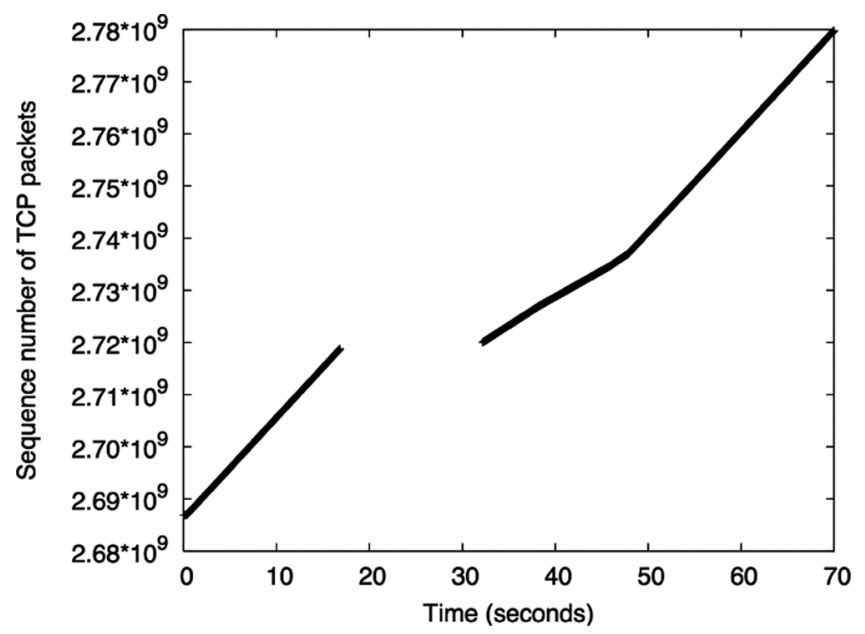




\subsection{Scalability studies}

The objective of our scalability studies is to explore how large an experiment can be supported by our routing infrastructure with the virtual router technique. The network model for this set of experiments consisted of multiple Autonomous Systems (ASes), each being an identical medium-size network called the campus network (shown in Figure 9). The campuses were connected to form a ring-like topology. This campus network model was used by DARPA NMS program as the baseline network model for studying simulation performance (Nicol, 2008). In particular, the campus network consisted of 508 end hosts and 30 router nodes. We set the delay and bandwidth of all links between the routers to $10 \mathrm{~ms}$ and $100 \mathrm{Mbps}$, respectively. Each router node had one corresponding XORP virtual router running outside of PRIME. The virtual routers were configured to adopt OSPFv2 and BGP4 as IGP and EGP respectively. Each AS installed one border router acting as a BGP speaker and peering with two BGP speakers in neighbouring ASes. We scaled up the experiment by increasing the number of ASes.

Figure 9 A campus network represents one AS, where the router $R_{0}$ in $N e t_{0}$ acts as the border router of the AS

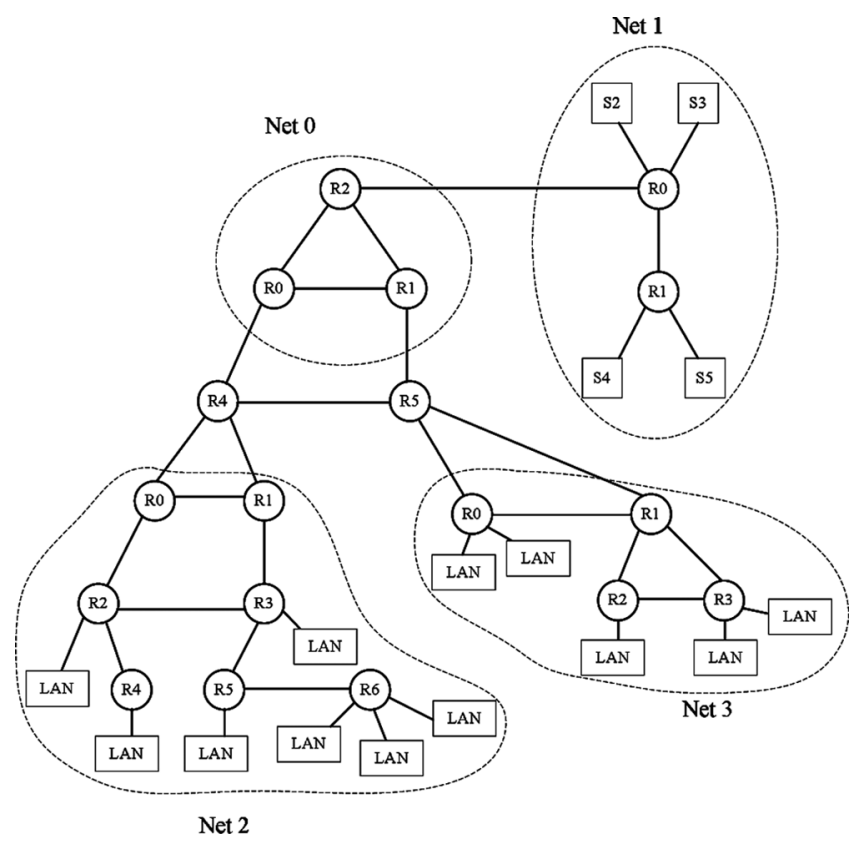

We used two DELL PowerEdge 1900 servers for this experiment, which were connected with a 100 Mbps Ethernet. Each machine had an Intel Quad-Core Xeon $2.0 \mathrm{GHz} \mathrm{CPU}$ and $8 \mathrm{~GB}$ memory. We set up OpenVZ environment on one machine, which hosted all the XORP virtual routers as well as the simulation gateway. PRIME ran on the other machine. We wrote a set of scripts to support automatic deployment of arbitrary network experiment scenarios. In particular, we started the XORP instances sequentially one after another using a script. In the case of eight campuses, the entire initialisation could take about $25 \mathrm{~min}$.
Table 2 shows the memory consumption on the XORP machine as we increased the number of virtual routers. The memory usage depends on two main factors: one is the fixed memory consumption associated with the initialisation of each virtual router, which leads to a cost proportional to the number of virtual routers included in the study; the other is the cost due to routing information maintained at each virtual router. The latter requires an $O\left(m \times p \times n^{2}\right)$ memory consumption, where $n$ is the number of virtual routers involved in the study, $p$ is the average number of prefixes associated with each virtual router, and $m$ is the memory consumed per prefix in the XORP RIB. As a result of these two factors, we observe a super-linear increase in the memory consumption.

Table 2 Memory usage (in MB) by XORP instances

\begin{tabular}{lccccc}
\hline$\#$ VRs & 30 & 90 & 150 & 210 & 240 \\
\hline Memory & 385.6 & 1570.6 & 3640.6 & 6295.6 & 7879.9 \\
\hline
\end{tabular}

Figure 10 shows the number of OSPF and BGP packets which passed through the routing infrastructure in an experiment consisting of eight campuses, i.e., with 240 virtual routers. The sampling interval was $10 \mathrm{~s}$. We can clearly observe the periodic OSPF link state refreshing behaviour that occurred at 30-min intervals (with significantly higher traffic through the routing infrastructure). In the default setting, OSPF requires that each Link State Advertisement (LSA) be refreshed every $30 \mathrm{~min}$. That is, the OSPF router that originates an LSA needs to increase the LSA's link state sequence number and re-flood the network every 1800 s. Since we started the routers sequentially during a period of approximately $25 \mathrm{~min}$, we observe that the impact from this refreshing behaviour was spread out accordingly. We used the default configuration for the BGP speakers that exported all local IP prefixes. Consequently, the OSPF link state refreshing triggered $\mathrm{BGP}$ updates to be sent to BGP peers. Thus, the BGP traffic fluctuated similarly with the OSPF traffic.

Figure 10 Routing packets seen by the emulation gateway with 240 XORP instances

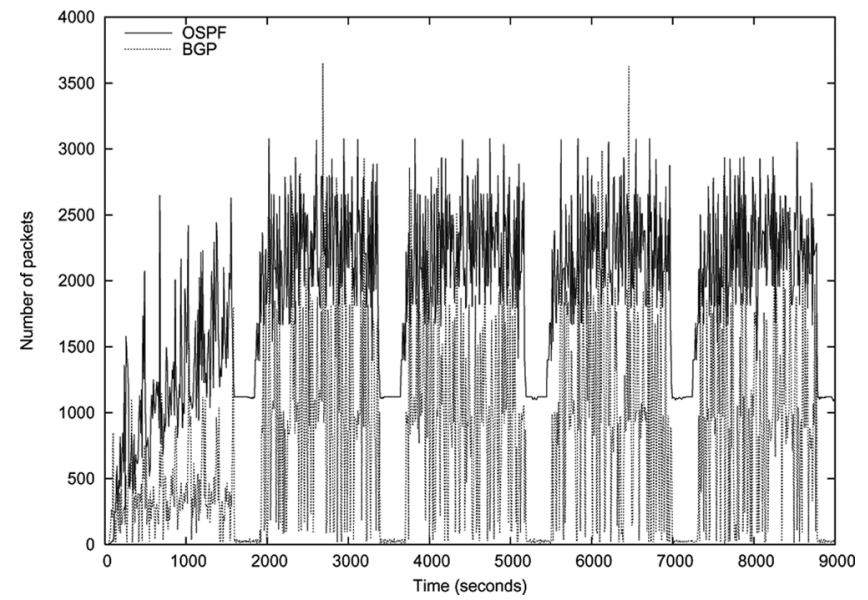


Figure 11 shows the CPU utilisation on the XORP machine in the same run. The CPU utilisation varied consistently with the behaviour of routing protocols. We notice that the peak CPU utilisation was less than $25 \%$. We can conclude that memory, instead of CPU, was the critical resource constraint for scaling up the experiment.

Figure 11 CPU utilisation with $240 \mathrm{XORP}$ instances

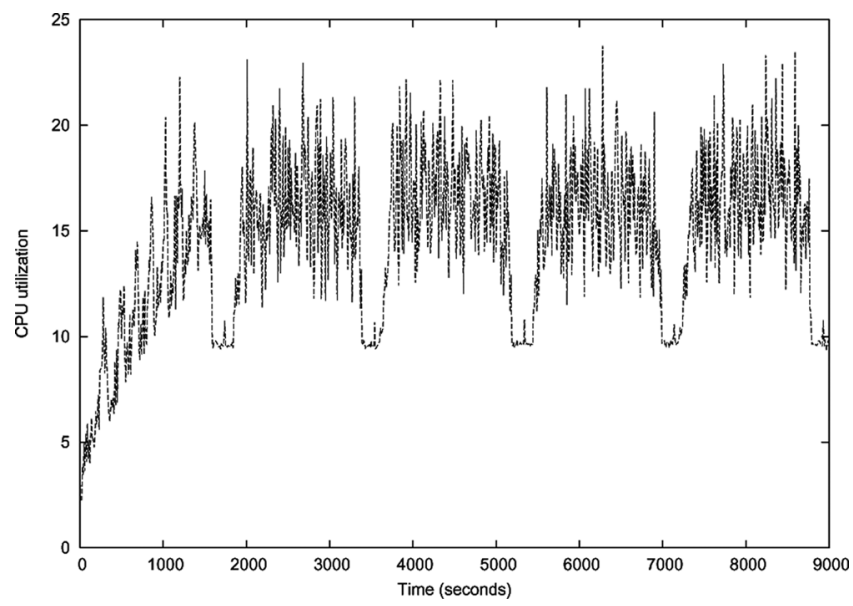

We also measured the latency of the emulation infrastructure, which is the major contributor to the latency overhead for synchronising forwarding information between PRIME and XORP instances. We enabled the LOOPBACK option in PRIME, in which case the reader thread immediately forwards the packets to the writer thread upon receiving them. Then we used ping to send 52-byte packets (i.e., the same size as the the forwarding information update packets) from one virtual router to another. Since the packets actually traverse the emulation infrastructure four times during a round trip, we can easily calculate the latency imposed by the emulation infrastructure. The result shows an average latency of $0.11 \mathrm{~ms}$, which confirms our belief that no significant forwarding skew between PRIME and XORP instances could occur with our forwarding plane offloading technique.

\section{Related work}

Most current real-time network simulators are based on existing network simulators added with the capabilities of interacting with real applications. Examples include NSE (Fall, 1999), IP-TNE (Bradford et al., 2000), MaSSF (Liu et al., 2003), and Maya (Zhou et al., 2004). NSE is an emulation extension of the popular $n s-2$ simulator (Breslau et al., 2000) augmented with the support for connecting with real applications and scheduling real-time events. IP-TNE is the first simulator we know that adopts parallel simulation techniques for large-scale network emulations. MaSSF is based on the DaSSF simulator (Liu and Nicol, 2008) with support for the grid computing environment. Maya is an emulation extension of the commercial QualNet simulator (Scalable Network Technologies, 2008) for wireless mobile networks. ROSENET (Gu and Fujimoto, 2007) is an emulation framework that separates network simulation that provides high-fidelity network resource estimates from low-fidelity emulation traffic forwarding. To some extent, this is similar in concept to our approach of separating routing and forwarding functions.

Other alternatives for conducting experimental network research are emulation and physical testbeds. EmuLab (White et al., 2002) is a notable emulation tool that consists of a large number of networked computers integrated and coordinated to present a virtual network environment. EmuLab currently supports hundreds of projects with thousands of users running over 18,000 experiments per year. PlanetLab (2008) is a collection of machines distributed across the internet and used by researchers to develop new network services. These machines run a customised operating system that allows the computing and networking resources to be shared among multiple experimentations that may take place simultaneously. A critical component of this type of physical testbeds is the live traffic.

Our work bears resemblance with VINI (Bavier et al., 2006). VINI is a virtual network infrastructure that enables realistic and controlled network experiment over PlanetLab. By using virtualisation technology, researchers are able to create a flexible virtual network topology. The implementation of VINI runs XORP instances within UML virtual machines and click for packet forwarding. We drew inspiration from the VINI design. Our scheme is different, however, in that we focus on enabling real network simulation to achieve realism, scalability, and flexibility. Our approach allows a large number of XORP instances beyond what can be supported by PlanetLab.

\section{Conclusions and future work}

A sound infrastructure allowing scalable routing experiments is a key enabler for the design and development of network routing protocols. We propose a novel infrastructure that combines a real-time network simulation engine with an emulated implementation of open-source XORP router software to provide a realistic, scalable, and flexible testbed for routing experiments. Offloading the forwarding plane from the XORP instances to the simulator allows scalability, by confining bulk traffic to be forwarded more efficiently within the simulator without exposing it to the external routers. An experimental evaluation of this infrastructure, with microbenchmark tests and a case study, demonstrates the effectiveness of our approach in minimising overhead and consequently improving accuracy and scalability. The scalability studies show that our routing infrastructure can support for a large scale routing experiment with as many as 240 virtual routers running on a single physical machine. We also report the memory is the bottleneck for multiplexing a large number of virtual routers by using virtualisation technology. 
Our current infrastructure for scalable routing experiments has limitations. For example, the synchronisation updates between the XORP protocol instances and their corresponding router nodes within PRIME are only one-directional in our current prototype implementation; updates are only propagated from the emulated XORP routers to the simulation engine. As immediate future work, we will allow information exchange in both directions so that we will be able to deal with more complex requirements in protocol operation.

\section{Acknowledgement}

This research is supported in part by National Science Foundation grants CNS-0546712, CNS-0836408, and HRD-0317692. We would like to thank Eric Johnson at FIU SCIS for the helpful discussions. We would also like to thank the anonymous reviewers for their constructive comments.

\section{References}

Abilene Network (2008) Abilene Observatory Summary Data View, http://abilene.internet2.edu/observatory/ data-views.html

Barham, P., Dragovic, B., Fraser, K., Hand, S., Harris, T., Ho, A., Neugebauer, R., Pratt, I. and Warfield, A. (2003) 'Xen and the art of virtualization', Proceedings of the 19th ACM Symposium on Operating Systems Principles (SOSP'03), Bolton Landing, New York, USA, pp.164-177.

Basu, A. and Riecke, J.G. (2001) 'Stability issues in OSPF routing', Proceedings of the ACM SIGCOMM Conference on Applications, Technologies, Architectures, and Protocols for Computer Communications (SIGCOMM'01), San Diego, California, USA, pp.225-236.

Bauer, D., Yuksel, M., Carothers, C. and Kalyanaraman, S. (2006) 'A case study in understanding OSPF and BGP interactions using efficient experiment design', Proceedings of the 20th Workshop on Principles of Advanced and Distributed Simulation (PADS'06), Washington, DC, USA, pp.158-165.

Bavier, A., Feamster, N., Huang, M., Peterson, L. and Rexford, J. (2006) 'In VINI veritas: realistic and controlled network experimentation', Proceedings of the ACM SIGCOMM Conference on Applications, Technologies, Architectures, and Protocols for Computer Communications (SIGCOMM'06), Pisa, Italy, pp.3-14.

Bhatia, S., Motiwala, M., Muhlbauer, W., Valancius, V., Bavier, A., Feamster, N., Peterson, L. and Rexford, J. (2008) Performance Evaluation of Virtualization Technologies for Server Consolidation, Technical Report GT-CS-07-10, Georgia Tech Computer Science, Atlanta, USA.

Bradford, R., Simmonds, R. and Unger, B. (2000) 'A parallel discrete event IP network emulator', Proceedings of the 8th International Symposium on Modeling, Analysis and Simulation of Computer and Telecommunication Systems (MASCOTS'00), pp.315-322.
Breslau, L., Estrin, D., Fall, K., Floyd, S., Heidemann, J., Helmy, A., Huang, P., McCanne, S., Varadhan, K., Xu, Y. and $\mathrm{Yu}, \mathrm{H}$. (2000) 'Advances in network simulation', IEEE Computer, Vol. 33, No. 5, pp.59-67.

Chim, T.W. and Yeung, K.L. (2004) 'Time-efficient algorithms for BGP route configuration', Proceedings of the IEEE International Conference on Communications (ICC'04), Paris, France, pp.1197-1201.

Cowie, J., Nicol, D. and Ogielski, A. (1999) 'Modeling the global internet', Computing in Science and Engineering, Vol. 1, No. 1, pp.42-50.

Dimitropoulos, X.A. and Riley, G.F. (2004) 'Large-scale simulation models of BGP', Proceedings of IEEE International Symposium on Modeling, Analysis, and Simulation of Computer and Telecommunication Systems (MASCOTS'04), Volendam, The Netherlands, pp.287-294.

Fall, K. (1999) 'Network emulation in the vint/ns simulator', Proceedings of the 4th IEEE Symposium on Computers and Communications (ISCC'99), pp.244-250.

Goodell, G., Aiello, W., Griffin, T.G., Ioannidis, J., McDaniel, P. and Rubin, A. (2003) 'Working around BGP: an incremental approach to improving security and accuracy of interdomain routing', Proceedings of the 10th Annual Network and Distributed System Security Symposium (NDSS'03), San Diego, California, USA, pp.150-161.

Gu, Y. and Fujimoto, R. (2007) 'Applying parallel and distributed simulation to remote network emulation', Proceedings for the 2007 Winter Simulation Conference, Washington DC, USA, pp.1328-1336.

Kohler, E., Morris, R., Chen, B., Jannotti, J. and Kaashoek, M.F. (2000) 'The click modular router', ACM Transactions on Compauter Systems, Vol. 18, No. 8, pp.263-297.

Liu, J. (2006) 'Packet-level integration of fluid TCP models in real-time network simulation', Proceedings of the 2006 Winter Simulation Conference (WSC'06), pp.2162-2169.

Liu, J. (2008) 'A primer for real-time simulation of large-scale networks', Proceedings of the 41st Annual Simulation Symposium (ANSS'08), Ottawa, Canada, pp.85-94.

Liu, J., Mann, S., Vorst, N.V. and Hellman, K. (2007) 'An open and scalable emulation infrastructure for large-scale real-time network simulations', Proceedings of the 26th Annual IEEE Conference on Computer Communications, Joint Conference of the IEEE Computer and Communications Societies (INFOCOM'07) MiniSymposium, pp.2476-2480.

Liu, J. and Nicol, D.M. (2008) Dartmouth Scalable Simulation Framework (DaSSF), http://www.cis.fiu.edu/ liux/ research/projects/dassf/index.html

Liu, X. and Chien, A.A. (2004) 'Realistic large-scale online network simulation', Proceedings of the SuperComputing Conference (SC'04), Pittsburgh, USA, pp.18-31.

Liu, X., Xia, H. and Chien, A.A. (2003) 'Network emulation tools for modeling grid behavior', Proceedings of 3rd IEEE/ACM International Symposium on Cluster Computing and the Grid (CCGrid'03), Tokyo, Japan, pp.18-26.

Mahajan, R., Wetherall, D. and Anderson, T. (2002) 'Understanding BGP misconfiguration', Proceedings of the ACM SIGCOMM Conference on Applications, Technologies, Architectures, and Protocols for Computer Communications (SIGCOMM'02), Pittsburgh, Pennsylvania, USA, pp.3-16. 
Nicol, D. (2008) DARPA NMS Baseline Network Topology, http://www.ssfnet.org/Exchange/gallery/baseline/index. html

Nordstrom, O. and Dovrolis, C. (2004) 'Beware of BGP attacks', ACM Computer Communications Review, Vol. 34, No. 2, pp.1-8.

Obradovic, D. (2002) 'Real-time model and convergence time of BGP', Proceedings of the 21st Annual Joint Conference of the IEEE Computer and Communications Societies (INFOCOM'02), New York City, USA, pp.893-901.

OpenVZ (2008) OpenVZ, http://openvZ.org/

Padala, P., Zhu, X., Wang, Z., Singhal, S. and Shin, K.G. (2007) Performance Evaluation of Virtualization Technologies for Server Consolidation, Technical Report HPL-2007-59, HP Laboratories.

PlanetLab (2008) Planetlab: An Open Platorm for Developing, Deploying, and Accessing Planetary-scale Services, http://www.planet-lab.org/

PRIME (2008) http:/ /www.cis.fiu.edu/prime/

Quagga (2008) Quagga Routing Suite, http://www.quagga.net/

Scalable Network Technologies (2008) http://scalablenetworks. com/

Shaikh, A., Dube, R. and Varma, A. (2002) 'Avoiding instability during graceful shutdown of OSPF', Proceedings of the 21st Annual Joint Conference of the IEEE Computer and Communications Societies (INFOCOM'02), New York City, USA, pp.883-892.

Shaikh, A. and Greenberg, A. (2001) 'Experience in blackbox OSPF measurement', Proceedings of the SIGCOMM Internet Measurement Workshop, San Francisco, California, USA, pp.113-125.
Shaikh, A., Kalampoukas, L., Dube, R. and Varma, A. (2000) 'Routing stability in congested networks: experimentation and analysis', Proceedings of the ACM SIGCOMM Conference on Applications, Technologies, Architectures, and Protocols for Computer Communications (SIGCOMM'00), Stockholm, Sweden, pp.163-174.

Soltesz, S., Potzl, H., Fiuczynski, M.E., Bavier, A. and Peterson, L. (2007) 'Container-based operating system virtualization: a scalable, high-performance alternative to hypervisors', Proceedings of the 2nd ACM SIGOPS/EuroSys European Conference on Computer Systemsof (EuroSys'07), Lisboa, Portugal, pp.3-16.

VRF (2008) Linux Virtual Routing and Forwarding (VRF), http://sourceforge.net/projects/linux-vrf/

White, B., Lepreau, J., Stoller, L., Ricci, R., Guruprasad, S., Newbold, M., Hibler, M., Barb, C. and Joglekar, A. (2002) 'An integrated experimental environment for distributed systems and networks', Proceedings of the 5th Symposium on Operating Systems Design and Implementation (OSDI'02), pp.255-270.

XORP (2008) XORP, http://www.xorp.org/

Zebra (2008) GNU Zebra, http://www.zebra.org/

Zhou, J., Ji, Z., Takai, M. and Bagrodia, R. (2004) 'Maya: integrating hybrid network modeling to the physical world', ACM Transactions on Modeling and Computer Simulation, Vol. 14, No. 2, pp.149-169. 